\title{
Analysis of metabolic pathways associated with neurogenesis in the retina of OXYS rat - model of age-related macular degeneration
}

\author{
D.V. Telegina*, O.S. Kozhevnikova, N.G. Kolosova \\ Institute of Cytology and Genetics SB RAS, Novosibirsk, Russia \\ *e-mail: telegina@bionet.nsc.ru
}

Key words: retina, AMD, neurogenesis, OXYS rats, RNA-seq

Motivation and Aim: Age-related macular degeneration (AMD) is a complex, multifactorial neurodegenerative disease that represents the most common cause of irreversible blindness. In recent years, AMD has grown younger: late stages of the disease are detected in relatively young people. It is known that pathogenesis of AMD based on the age-related structural and functional changes in the retina but the mechanisms that trigger the conversion of the normal age-related changes to the pathological process as well as the background of early development of AMD remain unclear. De novo neurogenesis in the adult mammalian retina very limited. Thereby the structural and functional features formed during the period of its maturation and formation can have long-term effects on the further ontogenesis of the tissue. The aim of this project is to study the possible contribution of the changes/disturbances of the postnatal retinal neurogenesis in the early development of AMD on the model of this disease - senescenceaccelerated OXYS rats.

Methods and results: We used RNA-Seq to compare gene expression profiles of retinas of senescence-accelerated OXYS rats and age-matched control Wistar rats at the age of 20 days, which is the period of completion of postnatal development of the organ of vision data of the retina. We determined the metabolic pathways controlling processes of neurogenesis based on the analysis of the transcriptome between OXYS and Wistar rats. We obtained a list of $349 \mathrm{DE}$ genes (at pval < 0.01) associated with neurogenesis. Of them, 213 were downregulated, and 136 were upregulated. These genes involved in the 22 functional groups such as nervous system development, neurogenesis, neuron projection development, neuron migration, axon development, dendrite development and eye development (according DAVID databased, FDR $<0.05$, EASE $>1.3$ ). As for the KEGG pathway analysis, we found that in OXYS rats compared Wistar rats, the DE genes were associated with a Axon guidance (Efnb2, Rasa1, Nrp1 et al.), Hedgehog signaling pathway (Ptch1, Csnk1g3, Hhip et al.), Wnt signaling pathway (Fzd4, Wnt5a, Tcf7 et al.) and Notch signaling pathway (Dtx3, Notch2, Jag2 et al.) (FDR <0.05). Using GeneMANIA, we identified 8 hub DE genes with the node degree $>55$. Of them, 6 were downregulated (Col5a2, Itga1, Dab2, Prrx 1, Fzd2, Calu) and 2 were upregulated (Ptch1 and $D \lg 4$ ). These genes probably play essential roles in the relevant biological systems. Thereby, the OXYS rats is characterized by disturbance of neurogenesis in retina.

Conclusion: Our data proposed that changes in retina's development precede the appearance of the AMD signs in OXYS rats and probably that the disruption of neurogenesis of retina significantly affects AMD pathogenesis.

Acknowledgements: This work was supported by the RFBR according to the research project No. 18-315-00216. 